\title{
ELIMINATION OF CIRCULAR ROUTES IN SPATIAL DYNAMIC REGRESSION EQUATIONS
}

\author{
H.J. BLOMMESTEIN* \\ Twente University of Technology, 7500 AE Enschede, The Netherlands
}

Received April 1984, final version received June 1984

Spatial lag operators play an important role in such fields as biometrics, geography and spatial econometrics. Higher-order spatial interactions can be represented by powering spatial lag operators. The paper discusses the conditions under which higher-order spatial lag operators will contain circular routes. Next, it is demonstrated that valid causal inference with the help of spatial dynamic regression models, necessitates the elimination of circular routes. It is shown that the ML-search procedure proposed by Ord (1975) and generalized by Blommestein (1983a), will generate spurious results in case of circular routes. A ML-procedure to obtain non-spurious estimated parameters in general dynamic spatial regression models is outlined. In addition some recent proposed approximate ML-search procedures [see Blommestein (1984b)], based on the spectral decomposition of a matrix, are modified in a similar way. The last section demonstrates empirically the purport of the theoretical results derived in the paper.

\section{Introduction}

The linear temporal lag operator $L$ and the polynomial operator $\alpha(L)$ have been extensively used in econometrics, notably in distributed lag analysis [cf. Dhrymes (1981)], and in time series analysis [cf. Box and Jenkins (1976)]. ${ }^{1}$ In other fields such as spatial economics, geography, biometrics and regional science, the transformation $L_{\mathrm{s}}$ plays an important role.

Definition 1. Let $V_{R}$ be a $R$-dimensional vector space, $K=\left\{x_{1}, \ldots, x_{R}\right\}$ any basis of $V_{R}$, and $L_{s}$ a linear transformation on $V_{R}$. Since every vector is a linear combination of the $x_{r}$, we have $L_{s} x_{r}=\sum_{r 1} w_{r r 1} x_{r 1}$ for $r \in\{1, \ldots, R\}$. The

*The author is indebted to N. Koper (Department of Electronic Engineering, Twente University of Technology) for computational assistance

${ }^{1}$ The transformation $L$ with properties summarized in definition $(i)$, is called a temporal lag operator [cf. Dhrymes (1981)]. Definition (i): Let $X$ be a set of all functions $x: N \rightarrow R$, where $\boldsymbol{N}=\{0, \pm 1, \pm 2, \ldots\}$ and $\boldsymbol{R}$ is the one dimensional Euclidean space. The transformation $L$ is defined on the vector space $X$ as $L x(t)=x(t-1), x \in X$. Since this is a linear transformation, $L$ is said to be a linear temporal lag operator. The powers of this operator, $L^{k}, k \in\{0,1,2, \ldots\}$ yield a set $L_{\tau}=\left\{L^{0}, L^{1}, L^{2}, \ldots\right\}$. The set of all finite linear combinations of elements of $L_{\tau}$ over the field of real numbers is a vector space, the vectors being polynomials in the temporal lag operator $L$. Definition (ii): The temporal polynomial operator of degree $M$ is defined as $\alpha(L)=\sum_{j=1}^{M} \alpha_{j} L^{j}$. 
set $\left\{w_{r r 1}\right\}$ of $R^{2}$ scalars defines the spatial operator $L_{s}$ :

$$
L_{s}=\left[\begin{array}{cccc}
w_{11} & w_{12} & \ldots & w_{1 R} \\
\vdots & \vdots & & \vdots \\
w_{R 1} & w_{R 2} & \ldots & w_{R R}
\end{array}\right]
$$

Remark. The set $\left\{w_{r r 1}\right\}$ is equivalent to the matrix of $L_{s}$ in the coordinate system $\boldsymbol{K}$.

In Blommestein (1984b) 3 types or forms of the spatial lag operator $L_{s}$ were studied: (i) symmetric matrices, i.e., $L_{s}=L_{s}^{T}$, (iia) asymmetric matrices which are non-triangular and (iib) upper (lower) triangular matrices, i.e., $w_{r r 1}=0$ for $r>r^{1}\left(r<r^{1}\right)$.

Definition 2. All three forms are assumed to be real non-negative $(R \times R)$ matrices $L_{s}=\left[w_{r r 1}\right]$, i.e., $w_{r r 1} \geqq 0, \forall r \neq r^{1} \in\{1, \ldots, R\}$; further it is assumed here that $w_{r r}=0, \forall r \in\{1, \ldots, R\}$.

The specification of $L_{s}$ is dependent on the spatial (economic) phenomena to be analysed. For example, triangular $L_{s}$ can be used for the study of Christallerian or Löschian central-place systems, while more general spatial interactions - such as spatial competition models - can be presented by dynamic regression models with (a)symmetric spatial lag operators.

Higher-order spatial interactions can be presented by powering spatial lag operators, that is, $L_{s}^{p,}, p>1$. To facilitate the interpretation of the information contained in $L_{s}^{p}$, consider the incidence matrix $Q$, corresponding to $L_{s}$. Next, conceive system (1) as a network, and the non-zero entries of the matrix $Q$ as the vertices (nodes) of that network. If $Q$ is raised to a certain power ( $p \geqq 2$ ), then the off-diagonal elements $Q^{p}$ denote the total number of connecting paths (chains) between each pair of vertices $\left(x_{r}, x_{r_{1}}\right)$. In general some of these paths will be redundant, also referred to as circular routes. Now, we are in the position to define the problem studied in this paper in the form of the following proposition and corollary.

Proposition 1. The use of higher-order $(p>1)$ spatial lag operators in spatial dynamic regression models will - in general - lead to spurious results, due to circular routes.

Corollary 1. Valid causal inference with the help of, for example, spatial dynamic regression equations, necessitates the elimination of circular routes.

The paper is structured as follows: Section 2 presents both a formal definition of circular routes and a method to eliminate redundant paths. 
Further some useful general mathematical results for both triangular and non-triangular $L_{s}$ are given. In section 3 the problem of redundant paths in spatial dynamic regression equations, is studied. A ML-method to obtain non-spurious estimated parameters in general dynamic spatial regression models, is outlined. An illustration of the elimination of circular routes in spatial regression models is presented in section 4 .

\section{Elimination of redundant paths in spatial lag operators}

Definition 3. Let $r, r^{1}, r^{11}, \ldots$ be arbitrary indices from the index set $\{1,2, \ldots, R\}$ of matrix $L_{s}$. A sequence $\left(r, r_{1}, r_{2}, \ldots, r_{s-1}, r^{1}\right)$, for $s \geqq 1$ and $r_{0}=r$, from the index set $\{1,2, \ldots, R\}$ is said to form a chain of length $s$ (or, equivalently, a $s$-step path) between the vertices or ordered pair $\left(x_{r}, x_{r 1}\right)$ if $w_{r r_{1}} w_{r_{1} r_{2}} \ldots w_{r_{s-2} r_{s-1}} w_{r_{s-1} r_{1}}>0$. In case $r=r^{1}$ such a chain is called a cycle of length $s$ between $r$ and itself.

Definition 4. A redundant chain or path (or, cquivalently, circular route) is a chain in which at least one index number repeats in the sequence of indices.

Example. Construct an incidence matrix $Q$, by replacing all the positive entries of $L_{s}$ by ones. The $p$ th $(\geqq 1)$ power of $Q$, denoted by $Q^{p}=\left[q_{r r 1}^{p}\right]$, has as its $\left(r, r^{1}\right)$ entry the total number of redundant and non-redundant $p$-step paths (i.e., chains of length $p$ ) from $r$ to $r^{1}$. Hence, the sequence $\left(r, r_{1}, r_{2}, \ldots, r_{p-2}, r_{p-1}, r^{1}\right)$, corresponding to the matrix product $q_{r r 1}^{p}=$ $\sum q_{r_{1}} q_{r_{1} r_{2}} \ldots q_{r_{p-2} r_{p-1}} q_{r_{p-1}}{ }^{1}$, will contain redundant information in case of circular routes. For example, $\left(r, r_{1}, r_{2}, \ldots, r_{1}, r_{2}, \ldots, r_{p-2}, r_{p-1}, r^{1}\right)$.

Before stating general results on the occurrence of circular routes in powering $L_{s}^{p}(p \geqq 1)$, a few more concepts will be introduced [see e.g., Seneta (1973)].

Definition 5a. $\Lambda \mathrm{n}$ index $r$ is essential, if $r \rightarrow r^{1}$ and $r^{1} \rightarrow r$. That is, $r$ and $r^{1}$ communicate (corresponding to a positive entry in the matrices $L_{s}^{p}$ and $Q^{p}$ ) which will be denoted as $r \leftrightarrow r^{1}$. Essential classes are obtained by subdividing all essential indices (denoted by $E_{i}$ ) such a way that all the indices belonging to one class communicate, but cannot lead outside the class, i.e., $E_{i 1} \rightarrow E_{i 11}$, $\forall i^{1} \neq i^{11} \in\{1, \ldots, N\}$.

Definition $5 b$. If $r \rightarrow r^{1}$ but $r^{1} \rightarrow r$ for some $r^{1}$ (corresponding with a zero entry in the matrices $L_{s}^{p}$ and $Q^{p}$ ), then the index is called inessential. By subdividing all inessential indices which communicate with some index in such a way that all indices in a class communicate, are called inessential classes (denoted by $I_{j}$ ). 
Definition 5c. Inessential and essential classes are called self-communicating classes.

Example. Consider a spatial system with 8 spatial units (regions, cities, etc.). Information on the interaction between those spatial units, stored in the $(8 \times 8)$ matrix $L_{s}$, is given below in the corresponding incidence matrix $Q$,

$\begin{aligned} & 1 \\ & 2 \\ & 3 \\ & 4 \\ & 5 \\ & 6 \\ & 7 \\ & 8\end{aligned} \quad\left[\begin{array}{llllllll}0 & 1 & 0 & 0 & 0 & 0 & 0 & 0 \\ 1 & 0 & 1 & 0 & 0 & 1 & 0 & 0 \\ 0 & 0 & 0 & 0 & 0 & 1 & 0 & 0 \\ 0 & 0 & 0 & 0 & 0 & 0 & 0 & 1 \\ 0 & 0 & 1 & 0 & 0 & 0 & 0 & 0 \\ 0 & 0 & 1 & 0 & 0 & 0 & 0 & 0 \\ 0 & 1 & 0 & 0 & 1 & 0 & 0 & 0 \\ 0 & 0 & 0 & 1 & 0 & 0 & 0 & 0\end{array}\right]$.

The essential classes corresponding to system (2) are $E_{1}=\{3,6\}$ and $E_{2}=$ $\{4,8\}$, while the inessential classes are equal to $I_{1}=\{1,2\}, I_{2}=\{5\}$ and $I_{3}=\{7\}$.

Given the definitions introduced above the following results can easily be derived.

Lemma 1. All indices of triangular $L_{\text {s }}$ fall into inessential non-selfcommunicating classes.

Proof. In case $L_{s}$ is triangular we have, $r \rightarrow r^{1}$ and $r^{1} \rightarrow r$ for all $r^{1}$. Hence, all indices are inessential and it is not possible to construct inessential classes such that all indices in a class communicate. Q.E.D.

Remark $i$. In case of an upper triangular $L_{s}$ the last row of $L_{s}$ is equal to $W_{R .}=(0 \ldots 0)$. Thus $R$ is an index which leads to no index at all and is also called inessential.

Remark ii. The assertion that a triangular $L_{s}$ does not possess (an) essential class(es) of indices also follows from a contradiction with lemma 1.1 in Seneta (1973), which states that a $(R \times R)$ non-negative matrix with at least one positive entry in each row possesses at least one essential class of indices.

Theorem 1. (i) Powering (real) non-negative symmetric and asymmetric nontriangular $(R \times R)$ matrices $L_{s}^{p}(p>1)$, will, in general, lead to the occurrence of circular routes. 
(ii) Raising triangular $L_{s}$ to a certain power does not lead to redundant paths, i.e., $L_{s}^{p}(p>1)$ has no circular routes.

Proof. (i) Raising $L_{s}$ to higher powers $(p>1)$ means, in general, that the number of connecting paths (or chains) will increase with increasing $p$. This implies that more entries of $L_{s}^{p}$ will be positive (i.e., $w_{r r_{1}}^{p}>0$ ), while the number of redundant and non-redundant ( $p$-step) paths [denoted by the $\left(r, r^{1}\right)$ entry of the corresponding incidence matrix $\left.Q^{p}\right]$ increases as well.

(ii) Since $L_{s}^{p}$ is reducible if $L_{s}$ is reducible [cf. Lancaster (1969, Ex. 4, p. 281)] and noting that a triangular matrix $L_{s}$ is reducible (which, is readily established from Lemma 1), yields the desired result. Q.E.D.

It may be necessary (see Corollary 1) to eliminate the circular routes of a matrix $L_{s}^{p}(p>1)$. Ross and Harary (1952) have constructed an algorithm for eliminating redundant paths of any given length. After determining a $(R \times R)$ matrix $C_{p}$, entirely consisting of redundant $p$-step paths, the following formulac is used to obtain a matrix $W^{p}$ of non-redundant $p$-step paths, satisfying the requirements of a (row) stochastic matrix,

$$
W^{p}=\left[\left(L_{s}^{p}-C_{p}\right)-\Delta\left(L_{s}^{p}-C_{p}\right)\right] S_{p},
$$

where $\Delta(X)$ is the diagonal operator, i.e., a $(R \times R)$ matrix whose principal diagonal is that of the $(R \times R)$ matrix $X$ and zeros elsewhere; $S_{p}$ is a $(R \times R)$ scaling matrix such that all rows of $W^{p}$ sum to one.

Example. For $p=3$ we have

$$
W^{3}=\left[\left(L_{s}^{3}-C_{3}\right)-A\left(L_{s}^{3}-C_{3}\right)\right] S_{3},
$$

where $C_{3}=\left[L_{s} \Delta\left(L_{s}^{2}\right)+\Delta\left(L_{s}^{2}\right) L_{s}\right]-\tilde{M} ; \tilde{M}=L_{s} 区 L_{s}^{T}$ [ 区 denotes the elementwise matrix multiplication operator due to Hadamard; see Halmos (1942)].

We conclude this section with a lemma.

Lemma 2. The spatial lag operator $W^{p}$ as defined in eq. (3), is imprimitive.

Proof. By contradiction. Suppose $W^{p}(p \geqq 1)$ is primitive, then $W^{p}>0$, some $p$ [see e.g., Lancaster (1969)]. However, it is assumed that the diagonal elements of $L_{s}$ are equal to zero, i.e., $w_{r r}=0, \forall r \in\{1, \ldots, R\}$ (see Definition 2). Further the diagonal operator $\Delta(\cdot)$ in eq. (3) ensures, that $\Delta\left(W^{p}\right)$ is equal to a square zero matrix. Q.E.D.

Remark. It was assumed that $W^{p}$ is irreducible. 
In the next section, some of the results presented above, will be used for the analysis of dynamic spatial regression models.

\section{Elimination of circular routes in spatial dynamic regression models}

In Blommestein (1983b) spatial versions of two procedures for determining the dynamic structure in spatial regression models, viz. common factor analysis (COMFAC) and economic factor analysis (ECONFAC), are proposed. A major objective of both procedures is to mitigate- the risk of misspecification due to the exclusion of variables and or lags. For this reason both COMFAC and ECONFAC start with a fairly general model (i.e., the unrestricted model or maintained hypothesis), such that the 'true' model is nested within it. Suppose that this general model can be expressed as a class of linear spatial dynamic regression equations,

$$
\gamma\left(L_{s}\right) \operatorname{vec} Z_{s}=E_{s}
$$

in which $\gamma\left(L_{s}\right)$ is a $(R \times(R(K+1)))$ matrix consisting of $K+1(R \times R)$ polynomial matrices $\gamma_{j}\left(L_{s}^{n_{j}}\right)$ in $L_{s}^{n_{j}}$ of orders $n_{j}, j \in\{0,1, \ldots, K\} ; L_{s}^{n_{j}}$ is the $n_{j}$ th order spatial lag operator $Z_{s}=\left[Y X_{1} \ldots X_{k}\right]$ a $(R \times(K+1))$ matrix with spatial observations; $E_{s} \sim \mathrm{NID}\left(0, \sigma_{e_{s}}^{2}\right)$ a $(R \times 1)$ vector with white noise error terms; and 'vec' a vectorization operator. Both COMFAC and ECONFAC employ a sequential testing framework for reducing the order of dynamics in system (4) [i.e., imposing restrictions on $\gamma\left(L_{s}\right)$ ] for all $K+1$ variables separately or simultaneously, until a test statistic exceeds a chosen critical value [see Blommestein (1983b) for details].

The log-likelihood function for model (4) can be written as [see Blommestein (1983a)]

$$
l(Y)=(-R / 2) \ln \left(2 \pi \sigma_{E_{s}}^{2}\right)-\left(1 / 2 \sigma_{E_{s}}^{2}\right)[A Y-X \beta]^{T}[A Y-X \beta]+\ln |A|,
$$

where $|A|=\left|I_{R}-\rho_{1} L_{s}^{1}-\rho_{2} L_{s}^{2}-\ldots-\rho_{p} L_{s}^{p}\right|$ is the Jacobian of the transformation from $E_{s}$ to $Y$. Let $L_{s}^{1}$ have $\lambda_{1}, \ldots, \lambda_{R}$ as its eigenvalues. The characteristic polynomial of $A$ can then be written as [see e.g., Blommestein (1984b)]

$$
|A|=\prod_{r=1}^{R}\left(1-\rho_{1} \lambda_{r}-\rho_{2} \lambda_{r}^{2}-\ldots-\rho_{p} \lambda_{r}^{P}\right)
$$

Define $\omega=\sigma_{E_{s}}^{2} ; D=A Y ; Z=X^{T} X ;$ and $M=I_{R}-X Z^{-1} X^{T}$. Setting $\partial[l(Y)] / \partial \beta$ to zero gives

$$
\widehat{\beta}=Z^{-1} X^{T} D .
$$


Similarly, $\partial[l(Y)] / \partial \omega=0$ and using (7) yields

$$
\hat{\omega}=(1 / R) D^{T} M D \text {. }
$$

Substituting (7) and (8) in (5) gives an objective function of the form

$$
l(\rho, \hat{\beta}, \hat{\omega})=\mathrm{const}-(R / 2) \ln \hat{\omega}+\ln |A| .
$$

The value of $|\rho|<1$ that maximizes $l(\rho, \hat{\beta}, \hat{\omega})$ can be found by a direct search procedure, using expression (6) [see for details appendix $A$ in Blommestein (1983a)].

In case $L_{s}$ is non-triangular, expression (5), and thus expression (9), will contain circular routes (cf. Theorem 1). Consequently the ML-estimates $\hat{\rho}$ and $\hat{\beta}$ arc contaminated by redundant information. Using the procedure proposed by Ross and Harary [cf. expression (3)] yields lag operators $W^{p}$ with non-redundant $p$-step paths. Substituting $W^{p}$ in $A$ gives $\tilde{A}=\left[I_{R}-\right.$ $\left.\rho_{1} W^{1}-\rho_{2} W^{2}-\ldots-\rho_{p} W^{D}\right]$. However, it should be cmphasized that $W^{p}$ is not simply the matrix $W$ raised to the $p$ th power! For this reason expression (6) should appropriately be modified,

$$
|\tilde{A}|=\prod_{r=1}^{R}\left(1-\rho_{1} \Theta_{1 r}-\rho_{2} \Theta_{2 r}-\ldots-\rho_{p} \Theta_{p r}\right)
$$

where $\left\{\Theta_{n_{j} r} \mid r=1, \ldots, R\right\}$ is the spectrum of the matrix $W^{n_{j}}$ with eliminated redundant $n_{j}$-step paths (from $L_{s}^{n_{j}}$ ).

The ML-search procedure, using objective function (9), becomes computationally more demanding if $p$ (i.e., the highest order of the spatial lag operator) increases. For this reason approximate ML-procedures, based on the spectral decomposition of a matrix, were proposed in Blommestein (1984a).

Note first that the spatial lag operator $L_{s}$ ( $L_{s}$ non-triangular) can be decomposed as follows:

$$
L_{s}=\sum_{r=1}^{R} \lambda_{r} G_{r}
$$

where $\left\{\lambda_{r}\right\}$ is the set of eigenvalues or spectrum of $L_{s}$, and $G_{r}$ the spectral components. Powering (10) yields

$$
L_{s}^{n_{j}}=\sum_{r=1}^{R} \lambda_{r}^{n_{j}} G_{r}, \quad n_{j} \geqq 1 .
$$

Eq. (11a) can be used for a first approximation of $A$ and or $\ln |A|$. Suppose 
$L_{\mathrm{s}}^{n_{j}} \approx G_{1}, \forall n_{j} \geqq 1$, i.e., $\sum_{r=2}^{R} \lambda_{r}^{n_{j}} G_{r}$ is dropped from (11a), then $A$ from (5) can be approximated as $A \approx I_{R}-\psi G_{1},\left(\psi=\sum_{j=1}^{P} \rho_{j}\right)$ and $\ln |A|$ as

$$
\ln |A| \approx \ln \left|I_{R}-\psi G_{1}\right|=\sum_{r=1}^{R}\left[\ln \left(1-\psi \mu_{r}\right)\right]
$$

where $\left\{\mu_{r}\right\}$ is the spectrum of $G_{1}$. Noting that $G_{1}$ is idempotent and the rank of $G_{1}$ is equal to one, then $\mu_{1}=1, \mu_{2}=\mu_{3}=\ldots=\mu_{R}=0$. This means that (12) further simplifies to

$$
\ln |A| \approx \ln \left(1-\psi \mu_{1}\right)=\ln (1-\psi), \quad|\psi|<1 .
$$

The relative error of approximating $L_{s}^{p}$ by $G_{1}$ is $\lambda_{2}^{p}$. Suppose this error is considered too large. A reduction of the relative error can be obtained by taking $L_{\mathrm{s}}^{j} \approx G_{1}+\lambda_{2}^{j} G_{2}$, and so on.

However, in view of our discussion above on circular routes, (11a) will contain redundant information. Obviously, the spectral decomposition of a spatial lag operator $W^{n_{i}}$ with non-redundant $n_{j}$-step paths is equal to

$$
W^{n_{j}}=\sum_{r=1}^{R} \Theta_{n_{j} r} G_{n_{j} r}
$$

A first approximation of $\ln |\tilde{A}|$, using the first spectral component, is equal to expression (12'). Clearly, further approximations should be based on (11b); e.g., $W^{3} \approx G_{31}+\Theta_{32} G_{32}$.

The next section demonstrates empirically the purport of Proposition 1 and Corollary 1.

\section{Estimation of spatial dynamic regression equations}

Consider the following specifications, chosen in Blommestein (1984b) as the maintained hypothesis in a COMFAC analysis of some models selected by Cliff and Ord (1981) for a spatial econometric analysis of the Irish economy:

$$
Y=\delta_{0} l+\delta_{1} X_{1}+\delta_{2} L_{s}^{1} X_{1}+\delta_{3} L_{s}^{2} X_{1}+\delta_{4} L_{s}^{1} Y+\delta_{5} L_{s}^{2} Y+E,
$$

where $Y$ is a $(R \times 1)$ vector with observations on the percentages of gross agricultural output (in value terms) of each of the $R(=26)$ countries in Eire consumed by itself; $X_{1}$ is a $(R \times 1)$ vector with values of an index of arterial road accessibility [see Cliff and Ord (1981) for details]; $L_{s}^{1}$ is a first order $(R \times R)$ spatial lag operator, whereby each non-zero off-diagonal element represents interaction between a pair $\left(r, r^{1}\right)$ of counties and all the diagonal 
elements are zero; $E \sim \mathrm{NID}\left(0, \sigma_{e}^{2}\right)$, i.e., a $(R \times 1)$ vector with white noise error terms.

The value of $\rho=\left(\delta_{4} \delta_{5}\right)^{T}$ that maximizes the objective function $l(\rho, \hat{\beta}, \hat{\omega})$ [compare eq. (9)] is given in table 1 . The characteristic polynomial is equal to $\ln |A|=\sum_{r=1}^{R}\left[\ln \left(1-\delta_{4} \lambda_{r}-\delta_{5} \lambda_{r}^{2}\right)\right]$.

Table 1

Estimated spatial dynamic regression models.

\begin{tabular}{|c|c|c|c|c|c|c|c|}
\hline & \multicolumn{2}{|l|}{ Model (13) } & \multicolumn{2}{|c|}{ Model (13a) } & \multirow{2}{*}{$\begin{array}{l}\text { Model (13b) } \\
\text { ML }\end{array}$} & \multirow{2}{*}{$\begin{array}{l}\text { Approxi- } \\
\text { mation } \\
\left(12^{\prime}\right)\end{array}$} & \multirow{2}{*}{$\begin{array}{l}\text { Approxi- } \\
\text { mation } \\
\left(12^{\prime \prime}\right)\end{array}$} \\
\hline & ML & OLS & ML & OLS & & & \\
\hline$\delta_{0}$ & -17.32 & +8.79 & -13.39 & -7.61 & -20.42 & -15.97 & -19.05 \\
\hline$\delta_{1}$ & +0.0008 & +0.0023 & +0.0027 & +0.0025 & +0.0028 & +0.0027 & +0.0028 \\
\hline$\delta_{2}$ & -0.0067 & -0.0032 & -0.0034 & -0.0042 & -0.0027 & -0.0031 & -0.0027 \\
\hline$\delta_{3}$ & +0.11 & -0.0039 & +0.0049 & +0.0035 & +0.0074 & +0.0057 & +0.0064 \\
\hline$\delta_{4}$ & +0.59 & +0.098 & +0.46 & +0.67 & +0.66 & +0.64 & +0.60 \\
\hline$\delta_{5}$ & -0.11 & +1.74 & +0.04 & +0.34 & -0.75 & -0.08 & -0.19 \\
\hline
\end{tabular}

Since $L_{s}^{2}$ contains redundant information, the ML-estimates $\hat{\delta}=\left(\hat{\delta}_{0} \ldots \hat{\delta}_{5}\right)^{T}$ are not reliable. Meaningful results can be obtained by substituting $\tilde{A}$ and $\ln |\tilde{A}|$ in (9) for $A$ and $\ln |A|$ respectively. Estimation results, ${ }^{2}$ referred to as model (13a), are reported in table 1 . The differences are striking and are therefore a nice illustration of Proposition 1 and Corollary 1. To demonstrate the importance of the Jacobian in (5), and hence in (9), OLS estimates were also calculated, by setting $A=I_{R}$ ( $I_{R}$ is the identity matrix), $X=\left[\imath X_{1} L_{s}^{1} X_{1} L_{s}^{2} X_{1} L_{s}^{1} y L_{s}^{2} Y\right]$ and $\ln |A|=0$ in (5).

Similarly, OLS-results were obtained but now with eliminated circular routes [i.e., the OLS counterpart of model (13a)]. From the comparison of the results in table 1 it can be concluded that: (i) the inclusion of the Jacobian term in (5) and (9) is important to offset the inconsistency of the OLS-estimates, (ii) the presence of redundant information seriously affects both ML- and OLS-results. To show the importance of the use of the correct Jacobian term [i.e., without redundant paths; see $\left(6^{\prime}\right)$ ], ML-estimates were calculated, using $\tilde{A}$ in (9), but with Jacobian term $\ln |A|$ instead of $\ln |\tilde{A}|$. The results [referred to as model (13b); see table 1] speak for themselves.

Finally, approximate ML-estimates were calculated, using the first spectral component for approximating $\ln |\tilde{A}|\left[\sec \left(12^{\prime}\right)\right]$, i.e., $\ln |\tilde{A}| \approx \ln \left[1-\left(\delta_{4}+\delta_{5}\right)\right]$.

A reduction in the error of approximating $\ln |\tilde{A}|$ was obtained by using two spectral components:

$$
\ln |\tilde{A}| \approx \sum_{r=1}^{2} \ln \left[1-\left(\delta_{4} \Theta_{1 r}+\delta_{5} \Theta_{2 r}\right)\right] .
$$

${ }^{2}$ The lag operators $L_{s}^{p}$ and $W^{p}(p \geqq 1)$ satisfy the requirements of a (row) stochastic matrix. Hence the largest eigenvalue is equal to one in all calculations. 
These estimates depart considerably from the 'true' ML-estimates in model (13a). As has been noted in Blommestein (1984a) this is not very surprising, since the eigenvalues $\left\{\Theta_{n_{j 1}} \geqq \Theta_{n_{j 2} \ldots} \ldots \Theta_{n_{j 26}}\right\}$ decline very slowly in magnitude for approximating $\ln |\tilde{A}|$, implies that only a small part of the information contained in $W^{n_{j}}$ is captured.

\section{Concluding remarks}

This paper clearly demonstrates the importance of eliminating circular routes in spatial dynamic regression models. Failing to remove redundant paths from spatial lag operators will result in seriously distorted MLestimates. This in turn affects the outcomes of various research activities, using these contaminated estimation results, or (test) statistics calculated from them. For example, specification search procedures like COMFAC [see Blommestein (1983b)] may result in incorrect model choices. In case model (4), or nested versions thereof, are hypothetical representations of spatial causal economic relationships in quantitative form, spurious falsifications may occur. Etcetera.

\section{References}

Blommestein, H.J., 1983a, Reanalysis of an irregular lattice, in: R. Henn et al., eds., Methods of operations research (Anthenäum Printers, Königstein).

Blommestein, H.J., 1983b, Specification and estimation of spatial econometric models: A discussion of alternative strategies for spatial economic modelling, Regional Science and Urban Economics 13, 250-271.

Blommestein, H.J., 1984a, Approximations to ML-estimators in spatial dynamic regression models, submitted for publication.

Blommestein, H.J., 1984b, Mathematical properties and econometric applications of spatial lag operators, Paper presented at the 23rd Western Regional Science Conference, February 2225, 1984, Monterey, CA.

Box, G.E.P. and G.M. Jenkins, 1976, Time series analysis, forecasting and control (Holden Day, San Francisco, CA).

Cliff, A.D. and J.K. Ord, 1981, Spatial processes: Models and applications (Pion, London).

Dhrymes, P.J., 1981, Distributed lags: Problems of estimation and formulation (North-Holland, Amsterdam).

Halmos, P.R., 1942, Finite-dimensional vector spaces (Princeton University Press, Princeton, $\mathrm{NJ})$.

Lancaster, P., 1969, Theory of matrices (Academic Press, New York).

Ord, J.K., 1975, Estimation methods for models of spatial interaction, Journal of the American Statistical Association 70, 120-126.

Ross, I.C. and F. Harary, 1952, On the determination of redundancies in sociometric chains, Psychometrica 17, 195-208.

Seneta, E., 1973, Non-negative matrices (Allen and Unwin, London). 